$\xi=$

\title{
The Content of Biologically Active Substances in Phytobiotics Used for Agricultural Animals and Poultry
}

\author{
Elena Victorovna Ulrikh1*, Rafik Shamilovich Khaliullin, Irina Alexandrovna Ganieva, Ekaterina Aleksandrovna \\ Izhmulkina, Mikhail Nikolayevich Arzjutov
}

1The Kemerovo State Agricultural Institute, Markovtseva Street, 5, 650056, Kemerovo, Russia

\begin{abstract}
Medicinal plants are producers of many biologically active substances - compounds that can influence biological processes in animals; such compounds include cardiac glycosides, saponins, sterols, carotenoids, polyphenols, alkaloids, vitamins, quinones, as well as substances with specific aroma, taste and color. Objects of the research were the extracts of purple coneflower (Echinacea purpurea), maral root (Leuzea carthamoides), holy thistle (Silybum marianum) and marigold (Calendula officinalis) obtained by low-temperature water-ethanolic extraction followed by low-temperature vacuum drying of the extracts. The content of biologically active substances was determined in accordance with the standard methods of chemical analysis for phytobiotic substances. As shown by the results of the studies, phytobiotic substances contain ascorbic acid, polysaccharides, tannins, carotenoids, phytosterols, fructose and inulin. They also contain many flavonoids, which, presumably, efficiently alter the economic-useful qualities of agricultural animals and poultry. The phytobiotical feed additives may indirectly control the intestinal microflora, supporting the internal protective mechanisms of the animal organism. The use of phytobiotical feed additives in the diet of farm animals and poultry is rationally substantiated, and contributes to abandoning the use of antibiotics in the fodder.
\end{abstract}

Keywords: Phytobiotic substances, Medicinal plants, Useful qualities, Biologically active substances, Agricultural animals and poultry

\section{Introduction}

A biologically active substance should be understood as any chemical compound, its origin being in material, which is directly or indirectly able to influence living systems: humans, animals, representatives of the microscopic world, and even plants [7].

This concept may be applied to virtually any substance, since one way or another, most chemical compounds, regardless of their structures, may have impact on a living organism [8]. In this case, what distinguishes biologically active substances from other substances?

Most specialists agree that a biologically active substance should mean only a chemical compound that is capable of interacting with the living organism in very low concentration [1]. Thus, for example atropine alkaloid contained in belladonna, in an amount higher than 0.2 milligrams, can lead to partial blockage of the mcholinergic receptors of the nervous system [2].

Biologically active substances are widely used both in folk and traditional medicine [3]. Some of them are extracted from plants and are therefore of natural origin, and some are artificially synthesized, but fully reproduce the structure of their biological counterparts [5].

Medicinal plants are producers of many biologically active substances, i.e. compounds that can influence biological processes in animals; such compounds include cardiac glycosides, saponins, sterols, carotenoids, polyphenols, alkaloids, vitamins, quinones, as well as substances with specific aroma, taste and color [6].
Biologically active substances belong to the products of secondary metabolism, which are called secondary metabolites or secondary products of biosynthesis. Currently, there are over 100,000 secondary metabolites known, which are produced by medicinal plants [9]. Most of them are practically and economically important products, and are used in pharmacological, cosmetic and food industry [10].

Creating a technology for producing phytobiotic substances to be included into the diets with the aim to increase the productivity of animals by improving fodder properties and stimulating the growth is a promising direction of replacing fodder antibiotics, increasing the ecological values of animal products, and ensuring fodder safety. Currently phytobiotic substances have shown their effectiveness in maintaining stable and optimal state of the gastrointestinal tract and the metabolic processes in the organism of animals and poultry, their immune status, due to exposure to certain biologically active compounds contained therein.

Studying the biological effect of medicinal plant extracts in the organisms of the agricultural animals and poultry has been the subject of a significant number of scientific papers of foreign scientists. For example, paper [8] examines the impact of feeding the Boswellia serrata resin on the hematological, biochemical and immunological indicators of broiler chicken. It has been concluded that this resin may be considered safe and effective as a dietary supplement for broilers.

Khan, H. Zaneb, S. Masood (2015) studied the influence of adding powder of leaves of the Moringa oleifera plant on the intestine morphology of broiler chickens. The length of the villi in the small intestine and mucin excretion increased during the experiments. 
M. Radaelli, et al. (2016) studied the antimicrobial activity of six essential oils widely used in Brazil as a condiment against Clostridium perfringens strains. All oils showed bactericidal activity at minimal concentrations, except for anise oil, which was bacteriostatic.

Singh G. et al. (2016) evaluated variability of phenolic content along with the antioxidant, antimicrobial and cytotoxic potential of certain traditional medicinal plants of India. The results showed that the selected traditional medicinal plants had antioxidant and antimicrobial effect.

Franciosini M.P. et al. (2016) studied the effect of aqueous extracts of common origanum (Origanum vulgare L.) and rosemary (Rosmarinus officinalis L.) on the immunity and the microbial population of broiler chicken intestines. Based on the results, it has been concluded that extracts of these herbs improve broilers immunity, contribute to balancing intestinal flora required for digestion and for protection from enteropathogenic microorganisms.

Santi Devi Upadhaya, et al. (2016) studied the effect of a gel phytogenic additive on nutrients' digestibility, blood characteristics, and intestinal morphology of weaned piglets. In experimental animals, digestibility of dry matter and energy increased with increasing the dosages of feed additives. The length of villi in the jejunum and the ileum increased.

Mohiti-Asli Maziar \& Ghanaatparast-Rashti Moein (2017) compared the influence of two phytogenic mixes on the immune response in broilers. Adding oregano essential oil into the diet had a positive effect on the immune system of broiler chickens.

Review by B. Kiczorowska et al. (2017) indicates that herbal immunomodulatory feed additives improve the immune system functioning, have significant impact on animals and poultry health status, and improve production indicators.

T. Ahmed S. and Yang C.-J. (2017) studied the effect of Punica granatum L. (common omegranate) additive on the immune system, intestinal and fecal microbiocenosis, and emissions of odorous gases from the excreta of broiler chickens. The results showed that the additive improved the immune system and the microbial ecosystem in broilers intestines along with reduced emission from the litter.

The Internet portal www.allaboutfeed.net devoted to feeding animals contains reports about successful use of phytobiotic substances in feeding cattle (Boczonadi A. 2 key points to fresh cow profits; Aubert T. Dairy management: It's all in the feed), pigs (Willems E. Microencapsulated organic acids and phytogenics; Steiner T. Phytogenics - Digestibility is the key), poultry (Ramirez D. Butyrate and botanical: A good mix; Mueller A., Aumiller T., Dirk van der Klis J. Phytogenics for better gut health in poultry; Schieder C. Phytogenics for efficiency in broilers Pirgozliev V., Rose S.P., Bravo D. Phytonutrients alter energy partitioning in poultry: the link with nutrition.). Antioxidant effect of phytobiotic substances has been noted in the Internet publication of Basharat S. Antioxidative potential of phytogenics and article of Konca Y. and coauthors (Konca, Y. et al., 2014).

Friedman et al. (2002) studied the antimicrobial effects of 96 essential oils and 23 components (active substances) and demonstrated that cinnamic aldehyde, thymol, carvacrol and eugenol showed the strongest antibacterial activity against strains of E. coli, Salmonella enterica, and L. monocytogenes.

Juliani et al. (2009) pointed out the importance of essential oils' chemical structure, having found that the antimicrobial activity of eugenol was higher, compared to the activity of methyl-eugenol.

$\mathrm{Si}$ et al. (2006) showed that Lactobacillus plantarum, Lactobacillus acidophilus Bifidobacterium spp. demonstrated lower sensitivity to the effect of carvacrol, thymol and eugenol, compared to pathogenic bacteria (E. coli and $S$. typhimurium). Similar results were shown by Manzanila (2005), who performed the research on pigs and confirmed the positive effect of plant extracts (carvacrol and cinnamic aldehyde) on the number and the ratio of lactobacilli and enterobacteria in the small intestine.

The obtained results indicate the possibility of using essential oils for maintaining desirable intestinal microflora, or eubiosis.

The use of phytobiotic substances stimulates secretion of luminal fluids, normalizes eubiosis in the digestive tract of the host animal, has positive effect on the morphological characteristics of the mucous membrane in the gastrointestinal tract (due to increasing the length of the villi with decreasing the crypt depth), which directly affects nutrient absorption, and in the end, results in increased productivity.

Zhou et al. (2007) made an experiment, where for the first time the lowest concentration $(200,400$ and $400 \mathrm{mg} / \mathrm{kg}$, respectively) of cinnamic aldehyde, thymol and carvacrol were identified, their use (separately) having a significant inhibitory effect on growth of Salmonella typhimurium was determined.

The authors demonstrated the synergistic effect of the combined use of these active ingredients (cinnamic aldehyde/thymol, cinnamic aldehyde/carvacrol and thymol/carvacrol), compared to using them individually.

Michiels et al. (2009) used various combinations of thymol and carvacrol, and confirmed the synergistic effect of these combinations on the total number of anaerobic bacteria in the small intestine of piglets.

The "Liptosa premix expert" phytobiotic substance made in Spain contains sodium formate, ammonium propionate, propionic acid, formic acid, fatty acids, natural plant extracts (cinnamon, cloves, oregano).

"Liptosa Premix Expert" is particularly effective to fight Clostridia, Salmonella and Escherichia coli. Laboratory tests and industrial experiments have proven the synergistic effect of essential oils and organic acids used for inhibiting bacteria. Essential oils of thymol, carvacrol, eugenol are used as strong antiviral, anti-infectious, antibacterial and immunostimulating medications. The sensitivity of Clostridia, Salmonella, and Escherichia coli to thymol, carvacrol and oregano oil $(\mathrm{P}<0.05)$ in anaerobic conditions has been proven.

The "Digestarom 1317" phytobiotical additive is a combination of many spices, plant extracts and their essential oils that have complex influence on the appetite of animals and poultry. Thus, peppermint oil stimulates secretion of saliva and gastric fluid, and has antibacterial properties; the root of gentian stimulates secretion of hydrochloric acid in the stomach, stimulates production of enzymes; horsetail has anti-inflammatory effect on the mucous membrane; camomile stimulates glands, helps to improve absorption of nutrients; oak bark has effect on the villi of the mucous membrane, and helps with bowel disorders. Natural digestion stimulants activate glands of internal secretion, e.g., secretion of bile and enzymes. With that digestion of feed is optimized, and the amount of harmful products of metabolism is reduced.

Activity of the phytobiotical preparation "Bistrong® 510" depends on the aromatic substances, anise and glucuronic acid, saponins, thymol, borneol, carvacrol, which stimulate biocatalytic and enzymatic processes in the digestive tract of poultry.

Phytobiotic substance "Bistrong ${ }^{\circledR}$ 510" allows to replace fodder antibiotics, ensuring high digestibility and the use of essential nutrients in the fodder, and excellent preservation of poultry.

The mechanism of action is based on the synergy of several herbal substances that contain active substances that influence a specific 
category of animals upon mutual combination. Essential oils (microencapsulation) are used to improve secretion of digestive juices, increase the production of endogenous enzymes, and improve nitrogen preservation in the organisms of the poultry, and body weight gain. Acute substances stimulate the formation of digestive enzymes and the secretion of digestive juices, as well as blood circulation and metabolic processes in the intestines. Bitter substances increase secretion of digestive fluids at the level of the autonomic nervous system. Saponins improve resorption of nutrients $(\mathrm{P}, \mathrm{Ca})$, increase permeability of the intestinal walls (increase the intercellular space), and reduce urea decomposition into ammonia (due to reduced intensity nitrogen compounds' decomposition to ammonia, water and carbon dioxide), which results in a decreased level of ammonia in the feces, the room and the environment (less stress on the respiratory system) [4].

In Russia and neighboring countries, the effect of some medicinal plant extracts on the metabolism, the intestinal microbiocenosis and the immune status of farm animals and poultry has also been sufficiently studied.

Studies of the Ukrainian scientists A. V. Gunchak, V. M Gunchak, I. B. Ratio (2015) have established that plant extracts, when added to animal and poultry feed, or drunk with water, contribute to intensification of the metabolic processes in the organism. It has been shown that the advantage of herbal preparations over synthetic ones is in the presence of natural components that structurally resemble biologically active compounds of the organism or the metabolic products (metabolites), and are therefore easily digested and absorbed, have low toxicity and do not cause side effects. The majority of essential oils demonstrate antimicrobial effect by disrupting bacterial cell walls, denaturing and coagulating proteins by altering their permeability of the cytoplasmic membrane, causing disruption of mandatory cellular processes, such as electron transport, protein translocation, phosphorylation and other enzyme-dependent reactions. Scientists have proven the efficiency of immunostimulants derived from medicinal plants for activating key ways of the immune system and enhancing protective mechanisms of the organism. The research has shown that plant extracts may ensure proper balance in the system of antioxidant protection that is fundamental for protecting cells from the damaging effects of free radicals and products of lipid peroxidation.

This work was focused on studying the content of biologically active substances in the extracts of medicinal plants obtained by the method of low temperature water-and-ethanol extraction followed by low-temperature vacuum drying of the extracts, with the aim of creating highly-efficient phytobiotical feed additives for agriculture animals and poultry, which might be able to decrease the actual consumption of feed antibiotics.

\section{Objects and Methods of the Research}

Objects of the research were extracts (pharmaceutical substances) of purple coneflower (Echinacea purpurea), maral root (Leuzea carthamoides), holy thistle (Silybum marianum) and marigold (Calendula officinalis) obtained by low-temperature waterethanolic extraction followed by low-temperature vacuum drying of the extracts.

For the purpose of quantitative determination of flavonoids in the extracts of medicinal plants, the photoelectric chromometering method of analysis (PEC method) was used.

The PEC method is based on measuring the optical density of colored solutions obtained by the reaction of flavonoids with salts of metals or combination with salts of diazonium.

The raw material was preliminarily cleaned of chlorophyll with chloroform, alcohol extract of flavonoids was obtained, followed by the reaction of forming an azo dye with diazotized streptocide, and the optical density of the colored solution was measured using photoelectric chromometer. The content of total flavonoids was calculated by the calibration graph built according to the standard sample of rutin.

The measurement was performed using a KFK-3 at the wavelength of 200 to $400 \mathrm{~nm}$.

For the quantitative determination of alkaloids, the method of Mach and Liederley was used. It is based on the alkaloids' ability to settle down under the influence of solutions of silicotungstic acid with formation of complex salts. When the alkaloid sediment is burnt, the silicotungstic acid remains. This fact can be used to determine the amount of pure alkaloid [3].

The content of biologically active substances was determined in accordance with the standard methods of chemical analysis for medicinal plants [8].

\section{Results and Discussion}

Holy thistle is used to treat liver diseases (hepatitis, cirrhosis, toxic lesions). Medications made of the fruit of holy thistle improve formation and excretion of bile and the protective properties of the liver to infectious and various poisoning of agriculture animals and poultry. They have antioxidant activity, stimulate synthesis of proteins and phospholipids, stabilize membranes, and accelerate regeneration of liver cells [4].

The content of flavonoids in holy thistle is shown in Table 1

Table 1. The content of biologically active substances in the extract of holy thistle

\begin{tabular}{|l|c|c|}
\hline \multirow{2}{*}{ Component } & \multicolumn{2}{|c|}{ The content of the component, \% (flowers) } \\
\cline { 2 - 3 } & $\begin{array}{c}\text { The sample at natural } \\
\text { humidity }\end{array}$ & In terms of dry substance \\
\hline Flavonoids in terms of rutin & 3.42 & 3.8 \\
\hline
\end{tabular}

As shown by the results of the research, holy thistle contains flavonoids $(3.8 \%)$, which, presumably, efficiently influences the complex of economically useful qualities of agricultural animals and poultry.

Purple echinacea improves the immunity through activating protective immune cells (phagocytes), and is used for treating bacterial and viral diseases in animals. All parts of plant contain polysaccharides (heteroxylans, arabinogalactans), essential oil in the anthodes (up to $0.5 \%$ ), in the green (up to $0.35 \%$ ), in the roots (up to $0.23 \%$ ), enzymes, macro- (potassium, calcium) and microelements (selenium, cobalt, silver, molybdenum, zinc, manganese, etc.). The main components of essential oils are noncyclic sesquiterpenes. The roots also contain inulin (up to 6\%), glucose (7\%), glycosides (echino-glycoside), echinacoside, phytosterols, organic acids (palmitic, linoleic, cerotic), phenol carbonic acid (chicoric, ferulic, coumaric, caffeic), fatty oils, betaine $(0.1 \%)$, resins $(2 \%)$, polyamides and antioxidants (echinacin, echinolon) [4].

Therefore, the use of echinacea extracts in the diets of rabbits for increasing productivity and the quality of products is an important issue. 
The content of flavonoids and other biologically active substances in the extract of purple echinacea is shown in Table 2 .

Table 2. The content of biologically active substances in the extract of purple echinacea

\begin{tabular}{|l|c|c|}
\hline \multicolumn{2}{|c|}{ Component } & \multicolumn{3}{|c|}{ The content of the component, \% (flowers) } \\
\cline { 2 - 3 } & $\begin{array}{c}\text { The sample at natural } \\
\text { humidity }\end{array}$ & In terms of dry substance \\
\hline Total phenylpropanoids reduced to chicoric acid & 2.33 & 2.5 \\
\hline Flavonoids in terms of rutin & 0.4 & 0.42 \\
\hline
\end{tabular}

The extract of purple echinacea contains chicoric acid $(2.5 \%)$ and flavonoids $(0.42 \%)$. Such content of biologically active substances will, presumably, efficiently alter the economic-useful qualities of agricultural animals and poultry.

Calendula has anti-inflammatory, antiseptic and biligenic action on farm animals and poultry. The phytoncidal properties of the extract are due to the essential oil in the plant.

The flowers of calendula have antispasmodic action. By relaxing the smooth muscles of such organs as the stomach, the intestines and the liver of farm animals and poultry, the plant also stimulates the secretory activity, which contributes to enhancing bile production and secretion, and increases the secretory activity of the stomach.

Table 3. Content of biologically active substances in the extract of calendula

\begin{tabular}{|l|c|c|}
\hline \multirow{2}{*}{ Component } & \multicolumn{2}{|c|}{ The content of the component, \% (flowers) } \\
\cline { 2 - 3 } & $\begin{array}{c}\text { The sample at natural } \\
\text { humidity }\end{array}$ & In terms of dry substance \\
\hline Flavonoids in terms of rutin & 0.71 & 0.75 \\
\hline Carotenoids & 0.0084 & 0.0088 \\
\hline
\end{tabular}

As the table data show, the extract of calendula contains carotenoids $(0.0088 \%)$ and flavonoids $(1.0 \%)$. The optimum content of biologically active substances allows to assume that feeding calendula to broiler chicken and laying hens will efficiently influence the set of economic-useful qualities in agricultural animals and poultry.

Preparations of maral root have tonic, stimulant, bracing and stimulating effect. The phytoecdysones contained in the
The presence of large amounts of carotenoids and flavonoids in the flowers promotes the capillary restorative activity.

High content of carotinoids, flavonoids, ascorbic and organic acids in the flowers of calendula causes an increase in the metabolic function of the liver, which improves bile composition, decreases the concentration of bilirubin and cholesterol, increases the secretory and excretory function in farm animals and poultry. And combination of calendula with extracts of chamomile improves the biliary function, which helps to eliminate cholestasis in the gallbladder [4].

The content of flavonoids and other biologically active substances in the extract of calendula is shown in Table 3.

Table 4. The content of biologically active substances in maral root

\begin{tabular}{|l|c|c|}
\hline Component & \multicolumn{2}{|c|}{ The content of the component, \% (flowers) } \\
\cline { 2 - 3 } & $\begin{array}{c}\text { The sample at natural } \\
\text { humidity }\end{array}$ & In terms of dry substance \\
\hline Ecdysten & 0.45 & 0.6 \\
\hline Inulin & 11.97 & 12.86 \\
\hline Flavonoids in terms of rutin & 0.38 & 0.4 \\
\hline
\end{tabular}

\section{Flavonoids content in extracts of medicinal plants}

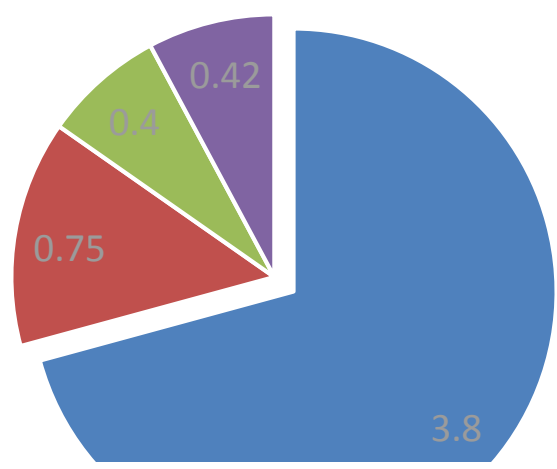

Fig. 1. Comparative data about the content of main biologically active substances in phytobiotic substances 
As the table data show, the extract of maral root contains Ecdysten $(0.6 \%)$, flavonoids $(0.4 \%)$, and Inulin $(12.86 \%)$. The optimum content of biologically active substances allows to assume that feeding maral root to cattle will efficiently influence the set of economic-useful qualities in agricultural animals.

The comparative data about the content of main biologically active substances in holy thistle, maral root, calendula and purple echinacea are shown in Figure 1. 1-Holy thistle; 2-Calendula officinalis; 3-Purple echinacea; 4-Maral root

Analysis of the data in Figure 1 shows that the greatest amount of flavonoids is contained in holy thistle, which results in its wide phytobiotical action.

Due to their multifunctional composition, phytobiotic substances added into the fodder of animals have a complex action, which has the positive effect on the productivity of animals, increases their appetite, improves digestibility of fodder, and increases the growth rate. Unlike antibiotics, this is not only due to the antimicrobial effect, but also due to the positive effect on the process of digestion (it increases secretion of saliva and gastric juices) with excretion of digestive enzymes. The better the nutrients are digested and absorbed in the small intestine of the animal, the less of them will get into the large intestine, which in turn will minimize the amount of nutrients required for the growth of bacterial flora in the large intestine.

The numerous studies performed at the cattle-breeding complexes and poultry farms of Russia and other countries show that the use of phytobiotics as a natural biologically active feed additive, including instead of antibiotics, has a positive effect on increasing animal productivity and reduces the cost of feed per unit of production.

Phytobiotical feed additives are introduced into about $5 \%$ of the total weight of fodder for farm animals worldwide every year. The main reasons that determine the use of phytogenic feed additives in animal husbandry are improvement of the diet digestibility, microbial efficiency, their use in accordance with the strategy of replacing feed antibiotics, and growth stimulation.

\section{Conclusion}

Limitations in the use of phytobiotic substances at Russian livestock enterprises are related to the absence of the prohibition to use feed antibiotics and high cost of imported phytobiotical feed additives. Due to the transition to the organic agriculture management (organic, biodynamic), there is the need to use alternatives to Russian feed antibiotics.

Thus, phytobiotical feed additives may indirectly control the intestinal microflora, supporting the internal protective mechanisms of the animal organism. The use of phytobiotical feed additives in the diet of farm animals and poultry is rationally substantiated, and contributes to abandoning the use of antibiotics in the fodder.

\section{Acknowledgments}

This article was prepared within the framework of the state agreement with the Ministry of Education and Science dated 03.10.2017 No. 14.610.21.0016 "Development and introduction of a new series of highly efficient phytobiotical feed supplements, based on the extracts of medicinal plants for transition to highly

productive and environmentally clean farming". The unique identifier of the project is RFMEF161017X0016.

\section{References}

[1] A. Calin-Sanchez, A. Figiel, K. Lech, Effects of Drying Methods on the Composition of Thyme (Thymus vulgaris L.) Essential Oil Drying technology, Vol. 31, Issue 2, 2013, pp. 224-235.

[2] A. Calin-Sanchez, K. Lech, A. Szumny, Volatile composition of sweet basil essential oil (Ocimum basilicum L.) as affected bydrying method. Food research international, Vol. 48, Issue 1, 2012, pp. 217-225.

[3] Sh. Dokhani, T. D. Durance, T. Cottrell, Drying Effects on Major Volatile and Phenolic Components of Achillea filipendulina Lam. Journal of essential oil bearing plants, Vol. 15, Issue 6, 2012, pp. 885-894.

[4] A. Ghasemzadeh, H. Z. E. Jaafar, A. Rahmat, Variation of the Phytochemical Constituents and Antioxidant Activities of Zingiber officinale var. rubrum Theilade Associated with Different Drying Methods and Polyphenol Oxidase Activity. Molecules. Vol. 21, Issue 6, 2016.

[5] Z. Kruma, R. Galoburda, M. Sabovics, G. Saravacos, P. Taoukis, M. Krokida, Aroma composition of microwave vacuum dried dill (Anethum graveolens L.) stems. 11th International Congress on Engineering and Food (ICEF); Athens, GREECE; May 22-26, 2011, pp. $1338-1343$.

[6] M. Robaina-Mesa, D. O. Lopez-Hernandez, E. Rodriguez-Chanfrau, J. Spray, Dried aqueous extract of Orthosiphon aristatus Blume (Java tea). Brazilian journal of pharmaceutical sciences, Vol. 53, Issue 3, No. UNSP e00015, 2017.

[7] M. Rubinskiene, P. Viskelis, E. Dambrauskiene, Effect of drying methods on the chemical composition and color of peppermint (Mentha x piperita L.) leaves. Zemdirbyste-agriculture. Vol. 102, Issue 2, 2015, pp. 223-228.

[8] M. Sledz, M. Nowacka, A. Wiktor, Selected chemical and physicochemical properties of microwave-convective driedherbs. Food and bioproducts processing, Vol. 91, Issue C4, 2013, pp. 421-428.

[9] M. Torki-Harchegani, D. Ghanbarian, A. G. Pirbalouti, Dehydration behaviour, mathematical modelling, energy efficiency and essential oil yield of peppermint leaves undergoing microwave and hot air. Renewable \& sustainable energy reviews, Vol. 58, 2016 pp. 407-418.

[10] E. Ulribe, D. Marti, A. Vega-Galves, Assessment of vacuum-dried peppermint (Mentha piperita L.) as a source of natural antioxidants. Food chemistry, Vol. 190, 2016, pp. 559-565. 